ANNALES

POLONICI MATHEMATICI

$89.2(2006)$

\title{
Collapse of warped submersions
}

\author{
by SzYMOn M. WaLcZaK (Łódź)
}

Abstract. We generalize the concept of warped manifold to Riemannian submersions $\pi: M \rightarrow B$ between two compact Riemannian manifolds $\left(M, g_{M}\right)$ and $\left(B, g_{B}\right)$ in the following way. If $f: B \rightarrow(0, \infty)$ is a smooth function on $B$ which is extended to a function $\tilde{f}=f \circ \pi$ constant along the fibres of $\pi$ then we define a new metric $g_{f}$ on $M$ by

$$
\left.\left.g_{f}\right|_{\mathcal{H} \times \mathcal{H}} \equiv g_{M}\right|_{\mathcal{H} \times \mathcal{H}},\left.\left.\quad g_{f}\right|_{\mathcal{V} \times T \widetilde{M}} \equiv \widetilde{f}^{2} g_{M}\right|_{\mathcal{V} \times T \widetilde{M}},
$$

where $\mathcal{H}$ and $\mathcal{V}$ denote the bundles of horizontal and vertical vectors. The manifold $\left(M, g_{f}\right)$ obtained that way is called a warped submersion. The function $f$ is called a warping function.

We show a necessary and sufficient condition for convergence of a sequence of warped submersions to the base $B$ in the Gromov-Hausdorff topology. Finally, we consider an example of a sequence of warped submersions which does not converge to its base.

\section{Introduction}

1.1. Riemannian submersion. Recall that a mapping $\pi: M \rightarrow B$ between two Riemannian manifolds $\left(M, g_{M}\right)$ and $\left(B, g_{B}\right), \operatorname{dim} B \leq \operatorname{dim} M$, is called a Riemannian submersion if it has maximal rank, and $g_{M}(u, w)=$ $g_{B}\left(\pi_{*} u, \pi_{*} w\right)$ for any vectors $u, w \in\left(\operatorname{Ker} \pi_{*}\right)^{\perp}$. We denote by $\mathcal{V}(x)=\operatorname{Ker} \pi_{* x}$ $\left(\mathcal{H}(x)=\left(\operatorname{Ker} \pi_{* x}\right)^{\perp}\right.$ resp.) the subspace of vertical (horizontal) vectors.

Lemma 1. Let $\pi: M \rightarrow B$ be a Riemannian submersion, where $M, B$ are compact Riemannian manifolds. The function $\widetilde{d}: B \ni x \mapsto \operatorname{diam}^{M}\left(\pi^{-1}(x)\right)$ is continuous.

Proof. Let $\varepsilon>0$ and $x_{0} \in B$. Since $\pi$ is continuous, there exist points $y_{1}, y_{2} \in \pi^{-1}\left(x_{0}\right)$ such that $d_{M}\left(y_{1}, y_{2}\right)=\operatorname{diam}^{M}\left(\pi^{-1}\left(x_{0}\right)\right)$.

Let $x \in B\left(x_{0}, \varepsilon / 2\right) \subset B$ and let $\gamma:[0, \delta] \rightarrow B, \delta>0$, be a geodesic curve such that $\gamma(0)=x, \gamma(\delta)=x_{0}, l(\gamma)=d_{B}\left(x, x_{0}\right)$. Denote by $\gamma_{i}, i=1,2$, the horizontal lifts of $\gamma$ such that $\gamma_{i}(\delta)=y_{i}$. It is clear that $l\left(\gamma_{i}\right)=l(\gamma)<\varepsilon / 2$.

2000 Mathematics Subject Classification: 53B21, 70G45.

Key words and phrases: Riemannian submersion, Gromov-Hausdorff topology, warped submersion. 
Hence

$$
\begin{aligned}
\operatorname{diam}^{M}\left(\pi^{-1}\left(x_{0}\right)\right) & =d_{M}\left(y_{1}, y_{2}\right) \\
& \leq l\left(\gamma_{1}\right)+d_{M}\left(\gamma_{1}(0), \gamma_{2}(0)\right)+l\left(\gamma_{2}\right) \\
& \leq \varepsilon+\operatorname{diam}^{M}\left(\pi^{-1}(x)\right) .
\end{aligned}
$$

In the same way we show that

$$
\operatorname{diam}^{M}\left(\pi^{-1}(x)\right) \leq \varepsilon+\operatorname{diam}^{M}\left(\pi^{-1}\left(x_{0}\right)\right) .
$$

Formulae (1) and (2) imply the continuity.

As a result, in further considerations we can assume that

$$
\operatorname{diam}^{M}\left(\pi^{-1}(z)\right) \leq 1
$$

for any $z \in B$.

1.2. Gromov-Hausdorff topology. The Gromov-Hausdorff distance between two compact metric spaces $\left(X, d_{X}\right)$ and $\left(Y, d_{Y}\right)$ is defined as (3) $\quad d_{\mathrm{GH}}(X, Y):=\inf \left\{\widetilde{d}_{H}(X, Y): \widetilde{d}\right.$ is an admissible metric on $\left.X \amalg Y\right\}$.

An admissible metric on $X \amalg Y$ is a metric that is an extension of $d_{X}$ and $d_{Y}$. Such a metric always exists, e.g.

$$
\begin{aligned}
& \left.\widetilde{d}\right|_{X \times X} \equiv d_{X},\left.\quad \widetilde{d}\right|_{Y \times Y} \equiv d_{Y}, \\
& \widetilde{d}(x, y)=\max \{\operatorname{diam}(X), \operatorname{diam}(Y)\}, \quad x \in X, y \in Y .
\end{aligned}
$$

In [1] it is shown that (3) defines a metric on the set of isometry classes of compact metric spaces. In further considerations we will need the following two facts.

LEMMA 2 (Gromov). If $\left(X, d_{X}\right)$ and $\left(Y, d_{Y}\right)$ are compact metric spaces and

$$
A=\left\{x_{1}, \ldots, x_{k}\right\} \subset X, \quad B=\left\{y_{1}, \ldots, y_{k}\right\} \subset Y
$$

are $\varepsilon$-nets on $X$ and $Y$, respectively, and if

$$
\left|d_{X}\left(x_{i}, x_{j}\right)-d_{Y}\left(y_{i}, y_{j}\right)\right| \leq \varepsilon, \quad 1 \leq i, j \leq k,
$$

then $d_{\mathrm{GH}}(X, Y) \leq 3 \varepsilon$.

A proof can be found in [3].

THEOREM 1. Let $\left(\left(X_{i}, d_{X_{i}}\right)\right)_{i \in \mathbb{N}},\left(Y, d_{Y}\right)$ be compact metric spaces. If $X_{i} \rightarrow Y$ in the Gromov-Hausdorff topology then for any $\eta>0$ and for any $\eta$-net $A=\left\{y_{1}, \ldots, y_{l}\right\}$ on $X$ there exists a sequence of $2 \eta$-nets $A^{i}=$ $\left\{x_{1}^{i}, \ldots, x_{l}^{i}\right\}$ on $X_{i}$ such that $A$ is a quasi-isometric limit of $A^{i}$, i.e. for any $j, k \in\{1, \ldots, l\}$,

$$
\left|d_{Y}\left(y_{j}, y_{k}\right)-d_{X_{i}}\left(x_{j}^{i}, x_{k}^{i}\right)\right| \rightarrow 0 \quad \text { as } i \rightarrow \infty .
$$

A proof can be found in [1]. 
1.3. Warped submersion. Let $\left(M, g_{M}\right),\left(B, g_{B}\right)$ be compact Riemannian manifolds, $\pi: M \rightarrow B$ a Riemannian submersion, and $f: B \rightarrow(0, \infty)$ a $C^{\infty}$-function on $B$. Then $\tilde{f}=f \circ \pi$ is a smooth function on $M$ constant along the fibres of $\pi$. Denote by $g_{f}$ the metric on $M$ given by

$$
\left.\left.g_{f}\right|_{\mathcal{H} \times \mathcal{H}} \equiv g_{M}\right|_{\mathcal{H} \times \mathcal{H}},\left.\left.\quad g_{f}\right|_{\mathcal{V} \times T \widetilde{M}} \equiv \widetilde{f}^{2} g_{M}\right|_{\mathcal{V} \times T \widetilde{M}}
$$

The manifold $M$ with metric $g_{f}$ will be called a warped submersion and denoted by $M_{f}$. The function $f$ will be called a warping function.

2. Main results. Let $\left(f_{n}: B \rightarrow(0, \infty)\right)_{n \in \mathbb{N}}$ be a sequence of smooth warping functions uniformly bounded on $B$ by a constant $C$. We ask what should be assumed about $\left(f_{n}\right)$ to ensure that the manifold $B$ is the limit of $M_{f_{n}}$ in the Gromov-Hausdorff topology.

Theorem 2. $M_{f_{n}} \rightarrow\left(B, g_{B}\right)$ in the Gromov-Hausdorff topology if and only if for any $\varepsilon>0$ there exists $N \in \mathbb{N}$ such that for all $n>N$ there exists an $\varepsilon$-net $A^{n} \subset B$ such that

$$
\left.f_{n}\right|_{A^{n}}<\varepsilon .
$$

Proof. $\Leftarrow$ Let $\eta>0$ and $n>N$. Let $A^{n}=\left\{y_{1}, \ldots, y_{k}\right\}$ be an $\eta$-net on $B$ such that $\left.f_{n}\right|_{A^{n}}<\eta$. Select points $x_{i} \in M_{f_{n}}, i \in\{1, \ldots, k\}$, in such a way that $\pi\left(x_{i}\right)=y_{i}$. Note that the set $\left\{x_{i}\right\}_{i \in\{1, \ldots, k\}}$ is a $2 \eta$-net on $M_{f_{n}}$. Indeed, let $y \in M_{f_{n}}$. There exists $j \in\{1, \ldots, k\}$ such that $d_{B}\left(\pi(y), y_{j}\right)<\eta$. Let $\gamma:[0, \delta] \rightarrow B$ be a minimal geodesic curve joining $\pi(y)$ and $y_{j}$ and $\widetilde{\gamma}$ its horizontal lift such that $\widetilde{\gamma}(0)=y$. We have

$$
d_{M_{f_{n}}}\left(y, x_{j}\right) \leq l(\widetilde{\gamma})+\operatorname{diam}^{M_{f_{n}}}\left(\pi^{-1}\left(y_{j}\right)\right) \leq l(\gamma)+\eta<2 \eta .
$$

Moreover, for all $i, j \in\{1, \ldots, k\}$,

$$
d_{B}\left(y_{i}, y_{j}\right) \leq d_{M_{f_{n}}}\left(x_{i}, x_{j}\right)
$$

Furthermore, if $\gamma:[0, \delta] \rightarrow B$ is a minimal geodesic curve joining $x_{i}$ to $x_{j}$ and $\widetilde{\gamma}$ its horizontal lift such that $\widetilde{\gamma}(0)=x_{i}$ then

$$
d_{M_{f_{n}}}\left(x_{i}, x_{j}\right) \leq l(\widetilde{\gamma})+d_{M_{f_{n}}}\left(\widetilde{\gamma}(\delta), x_{j}\right) \leq d_{B}\left(y_{i}, y_{j}\right)+\eta .
$$

Hence, from (4) and (5), $\left|d_{B}\left(y_{i}, y_{j}\right)-d_{M_{f_{n}}}\left(x_{i}, x_{j}\right)\right|<2 \eta$ for all $i, j \in$ $\{1, \ldots, k\}$. Lemma 2 gives us the statement.

$\Rightarrow$ Suppose that there exists $\varepsilon_{0}>0$ and a sequence $n_{k} \rightarrow \infty$ such that for any $k \in \mathbb{N}$ and any $\varepsilon_{0}$-net $A \subset B$ there exists $x \in A$ such that $f_{n_{k}}(x) \geq \varepsilon_{0}$. It is obvious that there exist $E_{0}>0$ and $y_{0} \in B$ such that $\left.f_{n_{k}}\right|_{B\left(y_{0}, E_{0}\right)} \geq \varepsilon_{0}$ for all $k \in \mathbb{N}$.

Now, suppose that $M_{f_{n_{k}}} \rightarrow B$ in the Gromov-Hausdorff topology. By Theorem 1, for any $\eta$-net $A=\left\{y_{1}, \ldots, y_{l}\right\} \subset B$ there exists a sequence of $2 \eta$-nets $A^{n_{k}}=\left\{x_{1}^{n_{k}}, \ldots, x_{l}^{n_{k}}\right\} \subset M_{f_{n_{k}}}$ such that $A$ is a quasi-isometric limit 
of $A^{n_{k}}$. Moreover, if $A$ is minimal and $\eta$ is small enough,

$$
\begin{aligned}
l \min \operatorname{vol}^{B} B(x, \eta / 4) & \leq \operatorname{vol} B, \\
l \max \operatorname{vol}^{M_{f_{n_{k}}}} B(x, 2 \eta) & \geq \operatorname{vol} M_{f_{n_{k}}} .
\end{aligned}
$$

Recall that for any compact manifold $\widetilde{M}$ there exists $\widetilde{\eta}>0$ and a constant $\widetilde{C} \geq 1$ such that for all $\eta<\widetilde{\eta}$ and $x \in \widetilde{M}$,

$$
\frac{1}{\widetilde{C}} \eta^{\operatorname{dim} \widetilde{M}} \leq \operatorname{vol} B(x, \eta) \leq \widetilde{C} \eta^{\operatorname{dim} \widetilde{M}}
$$

Hence, by (6) and (7),

$$
\begin{aligned}
0 & <\varepsilon_{0}^{\operatorname{dim} M-\operatorname{dim} B} \cdot \operatorname{vol}^{M} \pi^{-1}\left(B\left(y_{0}, E_{0}\right)\right) \leq \operatorname{vol} M_{f_{n}} \\
& \leq \operatorname{vol} B \frac{\operatorname{max~v^{2}} M_{f_{n_{k}}} B(x, 2 \eta)}{\min \operatorname{vol}^{B} B(x, \eta / 4)} \leq \operatorname{vol} B \frac{C_{M} C_{B} C^{\operatorname{dim} M-\operatorname{dim} B} \cdot(2 \eta)^{\operatorname{dim} M}}{(\eta / 4)^{\operatorname{dim} B}} .
\end{aligned}
$$

Hence $M_{f_{n_{k}}}$ cannot converge to $M$. This yields our statement.

3. Examples. Let $U \subset B$ be an open set and let $f: B \rightarrow[0, \infty)$ be a function such that $\left.f\right|_{U} \equiv 1$ and $\left.f\right|_{B \backslash U} \equiv 0$. Let $\left(f_{n}: B \rightarrow(0, \infty)\right)_{n \in \mathbb{N}}$ be a sequence of smooth functions on $B$ such that

$$
\left.f_{n}\right|_{U \backslash B(\partial U, 1 / n)} \equiv 1,\left.\quad f_{n}\right|_{B \backslash U} \equiv 1 / n, \quad f_{n} \leq 1 .
$$

It is obvious that $f_{n} \rightarrow f$. Moreover, the condition of Theorem 2 does not hold, so the limit of the sequence $M_{f_{n}}$ cannot be $B$. We then ask what the limit of $M_{f_{n}}$ is (if it exists).

Let $\sim$ be the equivalence relation on $M$ given by

$$
x \sim y \Leftrightarrow(\pi(x)=\pi(y) \text { and } \pi(x) \in B \backslash U) \text { or }(x=y \text { and } \pi(x) \in U)
$$

Let $\gamma_{x}^{y}:\left[0, \delta_{x}^{y}\right] \rightarrow B$ be a minimal geodesic curve joining $x, y \in B$. Let us set $X=M / \sim$ and define $\varrho: X \times X \rightarrow[0, \infty)$ as follows. If all $\gamma_{\pi(y)}^{\pi(x)}$ are contained in $U$ then

$$
\varrho(x, y)=\min \left\{\min _{z \in \partial U}\left\{d_{B}(\pi(x), z)+d_{B}(z, \pi(y))\right\}, d_{M}(x, y)\right\} ;
$$

if not,

$$
\varrho(x, y)=d_{B}(\pi(x), \pi(y)) .
$$

It is easy to show that $\varrho$ is a metric on $X$. This follows immediately from the fact that

$$
d_{M}(x, y) \geq d_{B}(\pi(x), \pi(y))
$$

and $d_{B}$ and $d_{M}$ are metrics on $B$ and $M$ respectively.

Now we can prove the following theorem. 
Theorem 3. $M_{f_{n}} \rightarrow(X, \varrho)$ as $n \rightarrow \infty$ in the Gromov-Hausdorff topology.

Proof. Let $\eta>0$ and let $E=\left\{x_{1}, \ldots, x_{k}, x_{k+1}, \ldots, x_{l}\right\}$ be an $\eta$-net on $X$ such that

$$
\left\{x_{1}, \ldots, x_{k}\right\} \subset \pi^{-1}(U) / \sim, \quad\left\{x_{k+1}, \ldots, x_{l}\right\} \subset \pi^{-1}(B \backslash U) / \sim .
$$

By (8) there exists $N \in \mathbb{N}$ such that for all $n>N$,

$$
\begin{aligned}
\operatorname{diam}_{f_{n}}\left([x]_{\sim}\right)<\eta & \text { for } x \in M \backslash \pi^{-1}(U), \\
\operatorname{diam}_{f_{n}}\left(\pi^{-1}\left(\pi\left(x_{j}\right)\right)\right)=1 & \text { for } j=1, \ldots, k .
\end{aligned}
$$

Let $n>N$ and let $E^{n}=\left\{y_{1}, \ldots, y_{l}\right\}$ be such that

$$
\left[y_{i}\right]_{\sim}=x_{i} \quad \text { for } i=1, \ldots, l .
$$

The set $E^{n}$ is a $2 \eta$-net on $M_{f_{n}}$. Indeed, let $y \in M_{f_{n}}$. There exists $j \in$ $\{1, \ldots, l\}$ such that $\varrho\left([y]_{\sim}, x_{j}\right)<\eta$. We consider the following cases:

1. $y \in \pi^{-1}(U)$ and $j \in\{1, \ldots, k\}$,

2. $y \in \pi^{-1}(U)$ and $j \in\{k+1, \ldots, l\}$,

3. $y \in \pi^{-1}(B \backslash U)$ and $j \in\{1, \ldots, k\}$,

4. $y \in \pi^{-1}(B \backslash U)$ and $j \in\{k+1, \ldots, k\}$.

We only handle the first case. The others are similar. Let $y \in \pi^{-1}(U), j \in$ $\{1, \ldots, k\}$. If any minimal geodesic curve $\gamma_{\pi\left(x_{j}\right)}^{\pi(y)} \subset U$ then, since $[y]_{\sim}=\{y\}$ and (9),

$$
\varrho\left([y]_{\sim}, x_{j}\right)=\min \left\{\min _{z \in \partial U}\left\{d_{B}(\pi(y), z)+d_{B}\left(z, \pi\left(x_{j}\right)\right)\right\}, d_{M}\left(y, x_{j}\right)\right\} .
$$

If $\varrho\left([y]_{\sim}, x_{j}\right)=d_{M}\left(y, x_{j}\right)$ then

$$
d_{M_{f_{n}}}\left(y, y_{j}\right) \leq d_{M}\left(y, y_{j}\right)=\varrho\left([y]_{\sim}, x_{j}\right)<2 \eta .
$$

Else if $\varrho\left([y]_{\sim}, x_{j}\right)=\min _{z \in \partial U}\left\{d_{B}(\pi(y), z)+d_{B}\left(z, \pi\left(x_{j}\right)\right)\right\}$ then

$$
\varrho\left([y]_{\sim}, x_{j}\right)=\min \left\{\min _{z \in \partial U}\left\{d_{B}\left(\pi\left([y]_{\sim}\right), z\right)+d_{B}\left(z, \pi\left(x_{j}\right)\right)\right\}\right.
$$

and for some $z_{0} \in \partial U$,

$$
\begin{aligned}
d_{M_{f_{n}}}\left(y, y_{j}\right) & \leq d_{B}\left(\pi(y), z_{0}\right)+d_{B}\left(z_{0}, \pi\left(y_{j}\right)\right)+\operatorname{diam}\left(\pi^{-1}\left(z_{0}\right)\right) \\
& =d_{B}\left(\pi\left([y]_{\sim}\right), z_{0}\right)+d_{B}\left(z_{0}, \pi\left(x_{j}\right)\right)+\eta \\
& =\varrho\left([y]_{\sim}, x_{j}\right)+\eta<2 \eta .
\end{aligned}
$$

Furthermore, for any $i, j \in\{1, \ldots, l\}$, we have

$$
\left|\varrho\left(x_{i}, x_{j}\right)-d_{M_{f_{n}}}\left(y_{i}, y_{j}\right)\right|<2 \eta \text {. }
$$

Indeed, if $k+1 \leq i \leq l, j \in\{1, \ldots, l\}$ then

$$
\varrho\left(x_{i}, x_{j}\right)=d_{B}\left(\pi\left(x_{i}\right), \pi\left(x_{j}\right)\right) \leq d_{M_{f_{n}}}\left(y_{i}, y_{j}\right)+\eta
$$


and as above

$$
\begin{aligned}
d_{M_{f_{n}}}\left(y_{i}, y_{j}\right) & \leq d_{M}\left(\pi\left(y_{i}\right), \pi\left(y_{j}\right)\right)+\operatorname{diam}^{M_{f_{n}}}\left(\pi^{-1}\left(\pi\left(y_{j}\right)\right)\right) \\
& \leq \varrho\left(x_{i}, x_{j}\right)+\eta
\end{aligned}
$$

Let $1<i, j \leq k$. Suppose that there exists a geodesic curve

$$
\gamma_{\pi\left(x_{j}\right)}^{\pi\left(x_{i}\right)}:\left[0, \delta_{\pi\left(x_{j}\right)}^{\pi\left(x_{i}\right)}\right] \rightarrow B
$$

not contained in $U$. Then $\varrho\left(x_{i}, x_{j}\right)=d_{B}\left(\pi\left(x_{i}\right), \pi\left(x_{j}\right)\right)$ and there exists $t_{0} \in$ $\left[0, \delta_{\pi\left(x_{j}\right)}^{\pi\left(x_{i}\right)}\right]$ such that $\gamma\left(t_{0}\right) \notin U$. Hence $\operatorname{diam}^{M_{f_{n}}}\left(\pi^{-1}\left(\gamma\left(t_{0}\right)\right)\right)<\eta$. Moreover,

$$
\varrho\left(x_{i}, x_{j}\right)=d_{B}\left(y_{i}, y_{j}\right) \leq d_{M_{f_{n}}}\left(y_{i}, y_{j}\right) \leq d_{M_{f_{n}}}\left(y_{i}, y_{j}\right)+\eta
$$

and

$$
\begin{aligned}
d_{M_{f_{n}}}\left(y_{i}, y_{j}\right) & \leq d_{B}\left(\pi\left(y_{i}\right), \gamma\left(t_{0}\right)\right)+d_{B}\left(\gamma\left(t_{0}\right), y_{j}\right)+\operatorname{diam}^{M_{f_{n}}} \pi^{-1}\left(\gamma\left(t_{0}\right)\right) \\
& \leq \varrho\left(x_{i}, x_{j}\right)+\eta
\end{aligned}
$$

Now, suppose that all minimal geodesics joining $\pi\left(y_{i}\right)$ to $\pi\left(y_{j}\right)$ are contained in $U$. If

$$
d_{M}\left(y_{i}, y_{j}\right)<\min _{z \in \partial U}\left\{d_{B}\left(\pi\left(y_{i}\right), z\right)+d_{B}\left(z, \pi\left(y_{j}\right)\right)\right\}
$$

then all minimal geodesic curves joining $y_{i}$ to $y_{j}$ in $M_{f_{n}}$ are totally embedded in $\pi^{-1}(U)$. Indeed, suppose by contradiction that there exists a minimal geodesic curve $\gamma_{0}:[0, \delta] \rightarrow M_{f_{n}}$ joining $y_{i}$ with $y_{j}$ which is not totally embedded in $\pi^{-1}(U)$. So there exist $x_{0} \in \pi^{-1}(\partial U)$ and $t_{0} \in(0, \delta)$ such $\gamma_{0}\left(t_{0}\right)=x_{0}$. We then have

$$
\begin{aligned}
d_{M_{f_{n}}}\left(y_{i}, y_{j}\right) & =l\left(\gamma_{0}\right)=\int_{0}^{\delta}\left\|\dot{\gamma}_{0}(t)\right\|_{M_{f_{n}}} d t \\
& =\int_{0}^{t_{0}}\left\|\dot{\gamma}_{0}(t)\right\|_{M_{f_{n}}} d t+\int_{t_{0}}^{\delta}\left\|\dot{\gamma}_{0}(t)\right\|_{M_{f_{n}}} d t \\
& \geq \int_{0}^{t_{0}}\left\|\left(\dot{\gamma}_{0}(t)\right)^{\perp}\right\|_{M_{f_{n}}} d t+\int_{t_{0}}^{\delta}\left\|\left(\dot{\gamma}_{0}(t)\right)^{\perp}\right\|_{M_{f_{n}}} d t \\
& \geq \int_{0}^{t_{0}}\left\|\pi_{*}\left(\dot{\gamma}_{0}(t)\right)^{\perp}\right\|_{B} d t+\int_{t_{0}}\left\|\pi_{*}\left(\dot{\gamma}_{0}(t)^{\perp}\right)\right\|_{B} d t \\
& \geq d_{B}\left(\pi\left(y_{i}\right), \pi\left(x_{0}\right)\right)+d_{B}\left(\pi\left(x_{0}\right), \pi\left(y_{j}\right)\right. \\
& \geq \min _{z \in \partial U}\left\{d_{B}\left(\pi\left(y_{i}\right), z\right)+d_{B}\left(z, \pi\left(y_{j}\right)\right\} .\right.
\end{aligned}
$$

But

$$
d_{M}\left(y_{i}, y_{j}\right) \geq d_{M_{f_{n}}}\left(y_{i}, y_{j}\right)
$$


So we get

$$
d_{M}\left(y_{i}, y_{j}\right) \geq \min _{z \in \partial U}\left\{d_{B}\left(\pi\left(y_{i}\right), z\right)+d_{B}\left(z, \pi\left(y_{j}\right)\right\}\right.
$$

which contradicts $(15)$.

Let $\gamma:[0, \delta] \rightarrow M_{f_{n}}$ be a minimal geodesic curve joining $y_{i}$ to $y_{j}$. Because all geodesic curves joining $y_{i}$ to $y_{j}$ are totally embeded in $\pi^{-1}(U)$,

$$
d_{M_{f_{n}}}\left(y_{i}, y_{j}\right)=\int_{0}^{\delta}\|\dot{\gamma}(t)\|_{M_{f_{n}}} d t=\int_{0}^{\delta}\|\dot{\gamma}(t)\|_{M} d t=d_{M}\left(y_{i}, y_{j}\right)=\varrho\left(x_{i}, x_{j}\right)
$$

Hence

$$
\left|d_{M_{f_{n}}}\left(y_{i}, y_{j}\right)-\varrho\left(x_{i}, x_{j}\right)\right|<\eta .
$$

Now, suppose that $d_{M}\left(y_{i}, y_{i}\right) \geq \min _{z \in \partial U}\left\{d_{B}\left(\pi\left(y_{i}\right), z\right)+d_{B}\left(z, \pi\left(y_{j}\right)\right\}\right.$. Let $z_{0} \in \partial U$ be a point at which $\min _{z \in \partial U}\left\{d_{B}\left(\pi\left(y_{i}\right), z\right)+d_{B}\left(z, \pi\left(y_{j}\right)\right\}\right.$ is achieved, and let

1. $\gamma_{1}:\left[0, \delta_{1}\right] \rightarrow B$ be a minimal geodesic curve joining $\pi\left(y_{i}\right)$ to $z_{0}$ and $\widetilde{\gamma}_{1}$ its horizontal lift such $\widetilde{\gamma}_{1}(0)=y_{i}$,

2. $\gamma_{2}:\left[0, \delta_{2}\right] \rightarrow B$ be a minimal geodesic curve joining $\pi\left(y_{j}\right)$ to $z_{0}$ and $\widetilde{\gamma}_{2}$ its horizontal lift such $\widetilde{\gamma}_{2}(0)=y_{j}$

3. $\gamma_{3}:\left[0, \delta_{3}\right] \rightarrow \pi^{-1}\left(z_{0}\right)$ be a minimal geodesic curve joining $\widetilde{\gamma}_{1}\left(\delta_{1}\right)$ to $\widetilde{\gamma}_{2}\left(\delta_{2}\right)$.

Let $\gamma:[0, \widetilde{\delta}] \rightarrow M_{f_{n}}, \widetilde{\delta}=\delta_{1}+\delta_{2}+\delta_{3}$, be given by $\gamma=\gamma_{2}^{-1} * \gamma_{3} * \gamma_{1}$. Then

$$
d_{M_{f_{n}}}\left(y_{i}, y_{j}\right) \leq l(\gamma)=\sum_{i=1}^{3} l\left(\gamma_{i}\right) \leq \varrho\left(x_{i}, x_{j}\right)+\eta
$$

On the other hand, if $\gamma:[0, \delta] \rightarrow M_{f_{n}}$ is a minimal geodesic curve from $y_{i}$ to $y_{j}$ then

1. if $\gamma([0, \delta]) \subset \pi^{-1}(U)$ then

$$
\begin{aligned}
\eta+d_{M_{f_{n}}}\left(y_{i}, y_{j}\right) & \geq l(\gamma)=\int_{0}^{\delta}\|\dot{\gamma}(t)\|_{M_{f_{n}}} d t=\int_{0}^{\delta}\|\dot{\gamma}(t)\|_{M} d t \\
& \geq \min _{z \in \partial U}\left\{d_{B}\left(\pi\left(y_{i}\right), z\right)+d_{B}\left(z, \pi\left(y_{j}\right)\right)\right\} \geq \varrho\left(x_{i}, x_{j}\right)
\end{aligned}
$$

2. if $\gamma([0, \delta]) \not \subset \pi^{-1}(U)$ then as in $(16)$,

$$
\eta+d_{M_{f_{n}}}\left(y_{i}, y_{j}\right) \geq \min _{z \in \partial U}\left\{d_{B}\left(\pi\left(y_{i}^{n}\right), z\right)+d_{B}\left(z, \pi\left(y_{j}^{n}\right)\right)\right\} \geq \varrho\left(x_{i}, x_{j}\right) .
$$

Hence by (11)-(14) and (17)-(20) we get (10).

Since $E^{n}$ and $E$ are $2 \eta$-nets on $M_{f_{n}}$ and $X$ respectively and for any $i, j \in\{1, \ldots, l\}$, we have

$$
\left|\varrho\left(x_{i}, x_{j}\right)-d_{M_{f_{n}}}\left(y_{i}, y_{j}\right)\right|<2 \eta
$$

Lemma 2 implies that $d_{\mathrm{GH}}\left(M_{f_{n}}, X\right)<6 \eta$. This yields our statement. 


\section{References}

[1] D. P. Chi and G. Yun, Gromov-Hausdorff Topology and its Applications to Riemannian Manifolds, Seoul National Univ., Seoul, 1998.

[2] M. Gromov, Metric Structures for Riemannian and Non-Riemannian Spaces, Birkhäuser, 1999.

[3] P. Petersen, Riemannian Geometry, Springer, 1997.

Faculty of Mathematics

University of Łódź

Banacha 22

90-238 Łódź, Poland

E-mail: sajmonw@math.uni.lodz.pl 